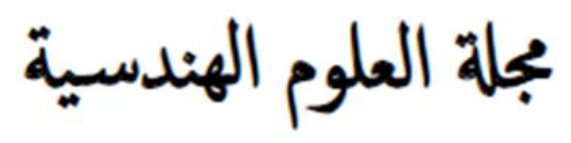

\title{
Phase-Field Modeling of Damage and Fracture in Laminated Unidirectional Fiber Reinforced Polymers
}

\author{
Aamir Dean ${ }^{1,6, *}$, Pavan Kumar ${ }^{2,6}$, Ammar Babiker ${ }^{3}$, Martin Brod ${ }^{1}$, Salih Elhadi Mohamed Ahmed ${ }^{4,5}$, Jose \\ Reinoso $^{6}$ and Elsadig Mahdi ${ }^{7}$

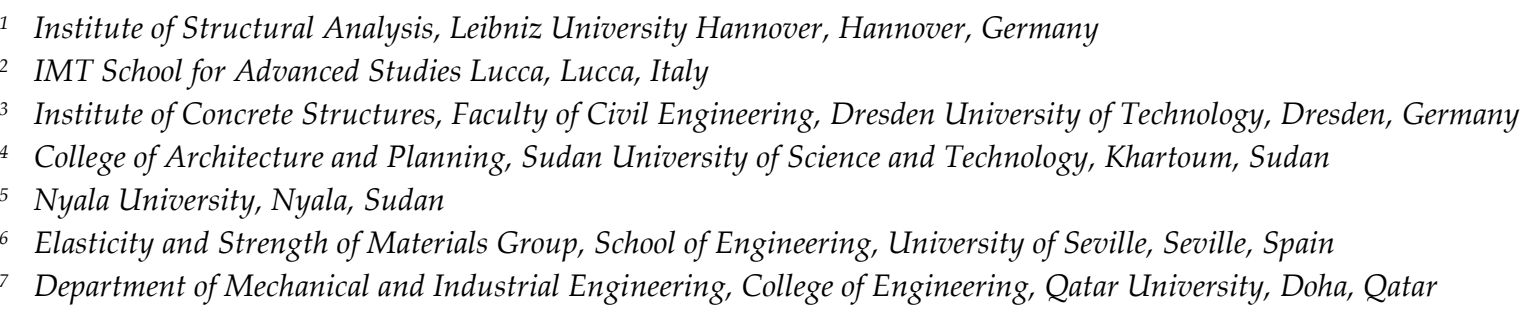

Article history: Received 15 May 2020, Received in revised form 4 November 2020, Accepted 11 November 2020

\begin{abstract}
The damage and fracture behavior of Fiber Reinforced Polymers (FRPs) is quite complex and is different than the failure behavior of the traditionally employed metals. There are various types of failure mechanisms that can develop during the service life of composite structures. Each of these mechanisms can initiate and propagate independently. However, in practice, they act synergistically and appear simultaneously. The difficulties that engineers face to understand and predict how these different failure mechanisms result in a structural failure enforce them to use high design safety factors and also increases the number of certification tests needed. Considering that the experimental investigations of composites can be limited, very expensive, and time-consuming, in this contribution the newly developed multi Phase-Field (PF) fracture model [1] is employed to numerically study the failure in different Unidirectional Fiber Reinforced Polymers (UFRPs) laminates, namely, fracture in single-edge notched laminated specimens, matrix cracking in cross-ply laminates, and delamination migration in multi-layered UFRPs. The formulation of the PF model incorporates two independent PF variables and length scales to differentiate between fiber and inter-fiber (matrix-dominated) failure mechanisms. The physically motivated failure criterion of Puck is integrated into the model to control the activation and evolution of the PF parameters. The corresponding governing equations in terms of variational formulation is implemented into the Finite Element (FE) code ABAQUS utilizing the user-defined subroutines UMAT and UEL.
\end{abstract}

Keywords: Fiber Reinforced Polymers, Damage and Fracture, Phase-Field Method, Finite Element Method

\section{INTRODUCTION}

Modern industry demands the advent and development of materials and structures with improved environmentally-friendly capacities that allow reducing carbon footprint, and at the same time with major safety performances with higher strengths and resistance to fatigue response, among many other attributes. The achievement of such conditions will contribute to the decrease in operational costs by virtue of the reduction of the required inspections and repairs. Within this context, recent advances in composites materials, more specifically Fiber Reinforced Polymers (FRPs), are helping to replace traditional materials across a host of engineering applications because of their versatility, enhanced durability and resistance to fatigue and corrosion, high strength-to-weight ratio, and lower maintenance and life-cycle costs 
[2], [3] Nevertheless, the full load-bearing capacity ability of composites has not yet been exploited.

With the continuously evolving trend of shifting to composites materials, there exists a recurrent need for a better understanding of their complex anisotropic, inhomogeneous, and inelastic behavior. The extensive understanding of damage and fracture events in FRPs is a matter of significant importance in many practical applications, with a strong interest in the wind turbine, automotive, aerospace, and aeronautical industries. There are various types of damage and fracture mechanisms that can develop during the service life of loaded composites structures. Although each of these failure mechanisms can initiate and evolve independently, in practice they act synergistically and appear simultaneously. The complexity in understanding and predicting how the failure mechanisms lead to a structural failure enforces the use of high design safety factors and increases the number of required certification tests.

The fact that experimental investigations of composites can be limited, very expensive, and time-consuming, has promoted the development of advanced and robust numerical modeling and simulation techniques to fully exploit the advantages of these materials under different loading conditions. The rapid growth of computational capacities motivates the development of a range of different sophisticated predictive models which allow the simulation of a wide variety of complex engineering problems.

However, the conventional theories of local Continuum Mechanics (CM), which are extensively used to trigger stiffness deterioration in FRPs, suffer from notable pathologies in the corresponding numerical implementation in conjunction with the fact that they present notable limitations for capturing well-known size effects. The alleviation of such drawbacks has been a matter of intensive research in the last two decades, as is the case of non-local Continuum Damage Mechanics (CDM) theories which inherently incorporate a characteristic length scale into the corresponding formulation.

Alternative routes for triggering fracture events are the strong discontinuity methodologies for fracture mechanics in solids, which incorporate an enriched kinematic description to model the material breakage upon loading, see [4]-[6] Despite recent developments in these methodologies, the numerical modeling of complex fracture problems remains a challenging issue, particularly in three-dimensional issues [7].

In the last decades, a potential methodology that can overcome the fundamental limitations of the other methodologies is the so-called Phase-Field (PF) method to fracture, see [8]-[11]. With strong roots in the energetic Griffith's vision of Fracture Mechanics (FM), PF methods endow the regularization of sharp crack discontinuities. However, this technique preserves the continuity of the displacement field, being especially suitable for triggering complex crack patterns.

Despite the relevant development of PF methods within the last decade, a careful revisitation of the State-of-the-Art shreds of evidence that these numerical techniques have been developed for their application for a limited type of engineering materials, with major attention for brittle fracture. However, PF methods possess enormous potential for the inclusion of phenomenological or physically-motivated failure criteria for brittle or ductile failure in a modular form, which can widen its range of application. Within this context, this research aims to develop sophisticated phenomenological material models based on the PF approach to fracture that can be employed into Finite Element Analysis (FEA) packages for virtual testing of damage and fracture in FRPs. A central aspect of this investigation is the development of a comprehensive theoretical and numerical study of PF methods for polymeric-based fiber reinforced composites, namely Unidirectional Fiber Reinforced Polymers (UFRPs), within the infinitesimal deformation setting.

\section{Multi PHASE-Field Formulation}

In this section, the multi PF formulation proposed in [1] for capturing intra-laminar (fiber and inter-fiber) failure mechanisms in UFRPs is briefly presented. The point of departure is the consideration of an arbitrary body in the general $\mathrm{n}_{\mathrm{dim}}$ Euclidean space, occupying the placemen 
$\mathcal{B} \in \mathbb{R}^{\mathbf{n}_{\mathrm{dim}}}$ with its external boundary $\partial \mathcal{B} \in \mathbb{R}^{\mathbf{n}_{\mathrm{dim}}-1}$ For any material point, the position vector is denoted by $\mathbf{x} \in \mathcal{B}$ The displacement field is identified by the vector $\mathbf{u}: \mathcal{B} \rightarrow \mathbb{R}^{\mathbf{n}_{\mathrm{dim}}}$, with infinitesimal stain tensor $\varepsilon:=\nabla^{\mathrm{sym}} \mathbf{u}$ for $\boldsymbol{\varepsilon}: \mathcal{B} \rightarrow \mathbb{R}^{\mathbf{n}_{\mathrm{dim}} \times \mathbf{n}_{\mathrm{dim}}}$. The displacement boundary conditions are prescribed as $\mathbf{u}=\overline{\mathbf{u}}$ on $\partial \mathcal{B}_{\mathbf{u}}$ and traction conditions are given by $\overline{\mathbf{t}}=\boldsymbol{\sigma} \cdot \mathbf{n}$ on $\partial \mathcal{B}_{\mathbf{t}}$ such that, kinematic and static boundary conditions satisfy: $\overline{\partial \mathcal{B}_{\mathrm{t}} \cup \partial \mathcal{B}_{\mathrm{u}}}=\partial \mathcal{B} \quad$ and $\partial \mathcal{B}_{\mathbf{t}} \cap \partial \mathcal{B}_{\mathbf{u}}=\varnothing$, where $\mathbf{n}$ is outward normal vector and $\sigma$ is the Cauchy stress tensor. Moreover, let $\Gamma_{d_{\mathrm{i}}}$ be a crack set incorporating the bulk cracks arising from the multi phase-field.

To account for multiple energies in the system, the total free energy functional describing the mechanics of body $\mathcal{B}$ is given as the sum total of internal and external energies acting on the system as follows, respectively:

$$
\Pi\left(\mathbf{u}, \Gamma_{d_{\mathrm{i}}}\right)=\Pi_{\mathrm{int}}\left(\mathbf{u}, \Gamma_{d_{\mathrm{i}}}\right)+\Pi_{\mathrm{ext}}(\mathbf{u})
$$

In classical Continuum Damage Mechanics CDM, the total internal energy is a state function of strain tensor $\varepsilon$ and internal damage like variable $d$ [1], [12], [13] The consistent generalization of the isotropic damage formulation for the consideration of different failure mechanisms can be postulated by the additive decomposition of the total internal energy into multiple contributions, in which each of them is associated with a certain failure mechanism. In such a postulation, a scalar damage variable $d_{\mathrm{i}}(\mathrm{i}=1, \ldots, \mathrm{n})$ is associated with each one of the $\mathrm{n}$ failure mechanisms, such that $d_{\mathrm{i}}=0$ for intact material state and $d_{\mathrm{i}}=1$ for fully broken state and so that $d_{\mathrm{i}} \in[0,1]$ for each $\mathrm{i}=1, \ldots, \mathrm{n}$. Moreover, to account for non-local damage evolution, the respective gradients $\nabla d_{i}$ are incorporated in the formulation. Herein, this additive decomposition postulation is applied within the context of PF approach to fracture for the prediction intra-laminar failure in UFRPs. Hence, the total internal energy is now an amalgamation of (i) total elastic energy constituting from bulk (fiber and inter-fiber) energy and (ii) surface energy (crack energy) stemming from bulk failure:

$\Pi_{\mathrm{int}}\left(\mathbf{u}, \Gamma_{d_{\mathrm{i}}}\right)=\Pi_{\mathrm{int}, \mathbf{F F}}\left(\mathbf{u}, \Gamma_{d_{\mathrm{FF}}}\right)+\Pi_{\mathrm{int}, \mathbf{I F F}}\left(\mathbf{u}, \Gamma_{d_{\mathrm{IFF}}}\right)$,

where

$$
\Pi_{\mathrm{int}, \mathbf{F F}}\left(\mathbf{u}, \Gamma_{d_{\mathrm{FF}}}\right)
$$

and

$\Pi_{\text {int,IFF }}\left(\mathbf{u}, \Gamma_{d_{\text {IFF }}}\right)$ correspond to the energies associated with fiber and inter-fiber, respectively.

With such decomposition at hand, the scheme herein used recalls that the dissipated energy arising from each of the individual failure mechanisms only affects their corresponding counterparts in the elasticity tensor.

The total energy of the fiber can be established as the sum total of the elastic energy and fracture energy associated with the fiber, respectively:

$$
\begin{aligned}
& \prod_{\mathrm{int}, \mathbf{F F}}\left(\mathbf{u}, \Gamma_{d_{\mathbf{F F}}}\right) \approx \Pi_{\mathrm{int}, \mathbf{F F}}\left(\mathbf{u}, d_{\mathbf{F F}}\right)=\int_{\mathcal{B}}\left(1-d_{\mathbf{F F}}\right) \Psi_{\mathbf{F F}}(\boldsymbol{\varepsilon}, \mathbf{A}) \mathbf{d} \Omega \\
& +\int_{\mathcal{B}} \mathfrak{G}_{\mathbf{c}, \mathbf{F F}}\left[\frac{1}{\int_{\mathbf{F F}}} d_{\mathbf{F F}}^{2}+\frac{l_{\mathbf{F F}}}{2}\left|\nabla d_{\mathbf{F F}}\right|^{2}\right] \mathbf{d} \Omega
\end{aligned}
$$

A denotes the so-called structural tensor which is characterizing the material inherent structure (anisotropy). $\Psi_{\mathbf{F F}}$ is the effective elastic energy contribution associated with the fiber. $\mathfrak{G}_{\mathbf{c}, \mathbf{F F}}$ is the fracture energy while $l_{\mathbf{F F}}$ is the material characteristic length associated with fiber failure:

$$
\begin{aligned}
& \prod_{\mathrm{int}, \mathbf{I F F}}\left(\mathbf{u}, \Gamma_{d_{\mathrm{IFF}}}\right) \approx \Pi_{\mathrm{int}, \mathbf{I F F}}\left(\mathbf{u}, d_{\mathbf{I F F}}\right)=\int_{\mathcal{B}}\left(1-d_{\mathrm{IFF}}\right) \Psi_{\mathbf{I F F}}(\boldsymbol{\varepsilon}, \mathbf{A}) \mathbf{d} \Omega \\
& +\int_{\mathcal{B}} \mathfrak{G}_{\mathbf{c}, \mathbf{I F F}}\left[\frac{1}{\ell_{\mathbf{I F F}}} d_{\mathbf{I F F}}^{2}+\frac{\digamma_{\mathbf{I F F}}}{2}\left|\nabla d_{\mathbf{I F F}}\right|^{2}\right] \mathbf{d} \Omega
\end{aligned}
$$

$\Psi_{\text {IFF }}$ is the effective elastic energy contribution associated with inter-fiber. $\mathfrak{G}_{\mathbf{c}, \mathbf{I F F}}$ is the fracture energy and $\tau_{\text {IFF }}$ is the material characteristic length associated with inter-fiber failure.

\section{VARIATIONAL FORMULATION AND STRONG FORM}

Relying on the considerations given in Section 2 , the total energy functional of the solid body $\mathcal{B}$, along with the cracks $\Gamma_{d_{\mathrm{i}}}$ at any arbitrary 
instance $t \in[0, T]$ takes the form:

$$
\Pi\left(\mathbf{u}, \Gamma_{d_{\mathrm{i}}}\right) \approx \Pi\left(\mathbf{u}, d_{\mathbf{i}}\right)=\Pi_{\mathrm{int}}\left(\mathbf{u}, d_{\mathbf{i}}\right)+\Pi_{\mathbf{e x t}}(\mathbf{u})
$$

where the internal and external contribution to the energy functional $\Pi\left(\mathbf{u}, d_{\mathbf{i}}\right)$ read, respectively:

$$
\begin{aligned}
& \Pi_{\mathrm{int}}\left(\mathbf{u}, d_{\mathbf{i}}\right)=\int_{\mathcal{B}}\left(1-d_{\mathbf{F F}}\right) \Psi_{\mathbf{F F}}(\varepsilon, \mathbf{A}) \mathbf{d} \Omega \\
& +\int_{\mathcal{B}} \mathfrak{G}_{\mathbf{c}, \mathbf{F F}}\left[\frac{1}{\ell_{\mathrm{FF}}} d_{\mathrm{FF}}^{2}+\frac{\ell_{\mathrm{FF}}}{2}\left|\nabla d_{\mathrm{FF}}\right|^{2}\right] \mathbf{d} \Omega \\
& +\int_{\mathcal{B}}\left(1-d_{\text {IFF }}\right) \Psi_{\text {IFF }}(\varepsilon, \mathbf{A}) \mathbf{d} \Omega \\
& +\int_{\mathcal{B}} \mathfrak{G}_{\mathbf{c}, \mathrm{IFF}}\left[\frac{1}{\ell_{\mathrm{IFF}}} d_{\mathrm{IFF}}^{2}+\frac{\ell_{\mathrm{IFF}}}{2}\left|\nabla d_{\mathrm{IFF}}\right|^{2}\right] \mathbf{d} \Omega \\
& \Pi_{\text {ext }}(\mathbf{u})=-\int_{\mathcal{B}} \mathbf{f}_{\mathbf{v}} \mathbf{d} \Omega-\int_{\partial \mathcal{B}}^{\overline{\mathbf{t}} \partial \mathbf{d} \Omega}
\end{aligned}
$$

where $\mathbf{f}_{v}$ is the deformation-independent volume-specific loads.

Following the standard Bubnov-Galerkin method, the three primary fields $\mathbf{u}, \delta_{\mathbf{F F}}$, and $d_{\text {IFF }}$ are extended by the corresponding test functions $\delta \mathbf{u}, \delta d_{\mathbf{F F}}$, and $\delta d_{\mathbf{I F F}}$, respectively. There accordingly, the weak form of the coupled displacement-crack phase-field problem is constructed by:

$$
\delta \Pi\left(\mathbf{u}, d_{\mathbf{i}}\right)=\delta \Pi_{\text {int }}\left(\mathbf{u}, d_{\mathbf{i}}\right)+\delta \Pi_{\text {ext }}(\mathbf{u})=0
$$

Moreover, after algebraic simplifications, the strong form of the field equations can be reduced to the following:

$$
\operatorname{div} \boldsymbol{\sigma}+\mathbf{f}_{\mathbf{v}}=0 \text { in } \mathcal{B} \text { and } \boldsymbol{\sigma} \cdot \mathbf{n}=\overline{\mathbf{t}} \text { on } \partial \mathcal{B}_{\mathbf{t}}
$$

$2\left(1-d_{\mathbf{F F}}\right) Q_{\mathbf{F F}} \mathcal{H}_{\mathbf{F F}}=\mathfrak{G}_{\mathbf{c}, \mathbf{F F}} \delta_{\mathbf{F F}} \gamma\left(d_{\mathbf{F F}}, \nabla d_{\mathbf{F F}}\right)$ in $\mathcal{B}$ and

$\nabla d_{\mathbf{F F}} \cdot \mathbf{n}=0$ on $\partial \mathcal{B}$

$2\left(1-d_{\text {IFF }}\right) Q_{\text {IFF }} \mathcal{H}_{\text {IFF }}=\mathfrak{G}_{\mathbf{c}, \mathbf{I F F}} \delta_{\text {IFF }} \gamma\left(d_{\text {IFF }}, \nabla d_{\text {IFF }}\right)$ in $\mathcal{B}$ and $\nabla d_{\text {IFF }} \cdot \mathbf{n}=0$ on $\partial \mathcal{B}$

where in the previous expressions $\operatorname{div}[\bullet]$ represents the divergence operator and $\gamma$ is the so-called crack density functional. The terms $\mathcal{H}_{\mathrm{FF}}$ and $\mathcal{H}_{\text {IFF }}$ are the crack driving forces related to fiber and inter-fiber failure, respectively. Herein, $Q_{\text {FF }}$ and $Q_{\text {IFF }}$ are activation flags for the current crack driving forces for fiber and inter-fiber failure, respectively, and are activated if and only if their respective Puck failure criterion has been met. In accordance with the Pucks failure criteria, the crack driving force of each $\mathrm{j}=\mathrm{FF}$, IFF are given by:

$\mathcal{H}_{\mathbf{j}}=\xi_{\mathbf{j}}\left[\left\langle\frac{\max _{\tau \in[0, \mathbf{t}]} \Psi_{\mathbf{j}}(\tau)}{\Psi_{\text {init } \mathbf{j}}}-1\right\rangle_{+}\right]$

where $\xi_{\mathbf{j}}$ is a dimensionless parameter that characterizes the damage activation and post-peak behaviors. $\Psi_{\text {init, }}$ is the effective elastic energy required for damage initiation for each of $j=F F$,IFF. For the description of Puck theory of failure, the reader is referred to [14].

The unilateral stationary condition of the total internal energy functional implies that $\delta \prod_{\text {int }}=0$ for all $\left(\delta \mathbf{u}, \delta d_{\mathbf{F F}}, \delta d_{\mathbf{I F F}}\right)>0$ and $\delta \Pi_{\text {int }}>0$ for $\left(\delta \mathbf{u}, \delta d_{\mathbf{F F}}, \delta d_{\mathbf{I F F}}\right)=0$ along with the irreversibility and boundedness of $\delta_{\mathbf{F F}}$ and $d_{\text {IFF }}$ leads to the first-order optimality (KKT) conditions for the quasi-static evolution [10], [15], [16].

The solution of the proposed coupled displacement multi phase-field fracture problem is obtained after discretizing the space using the Finite Element Method (FEM). The resulting non-linear system of equations is implemented in the finite element software ABAQUS. For this purpose, a user-defined UMAT is written for the solution of equilibrium equations associated with the displacement field whereas UEL is utilized for solving the fracture associated problem. The details of the finite element implementation are omitted here for the sake of brevity.

\section{APPLICATIONS}

In this section, the failure in different UFRPs laminates is studied employing the above-described formulation. 


\section{A. Material Properties}

Herein, the Unidirectional Carbon Fiber Reinforced Polymers (UCFRPs) IM7/8552 and Unidirectional Glass Fiber Reinforced Polymers (UGFRPs) E-Glass/MY750 are investigated. The elastic material properties of IM7/8552 and E-Glass/MY750 ply are shown in Table I and Table II, respectively, consistent with the experimental results in [17], [18].

TABLE I: IM7/8552: ELASTIC MATERIAL PROPERTIES

\begin{tabular}{lllll}
\hline \hline$E_{11}(\mathrm{GPa})$ & $E_{22}(\mathrm{GPa})$ & $G_{12}(\mathrm{GPa})$ & $v_{12}$ (minor) & $v_{23}$ \\
\hline 161.0 & 11.38 & 5.17 & 0.03 & 0.43 \\
\hline \hline
\end{tabular}

TABLE II: E-GLASS/MY750: ELASTIC MATERIAL PROPERTIES

\begin{tabular}{lllll}
\hline$E_{11}(\mathrm{GPa})$ & $E_{22}(\mathrm{GPa})$ & $G_{12}(\mathrm{GPa})$ & $v_{12}$ (minor) & $v_{23}$ \\
\hline 161.0 & 11.38 & 5.17 & 0.03 & 0.43 \\
\hline \hline
\end{tabular}

The fracture energy properties and phase-field parameters associated with IM7/8552 and E-Glass/MY750 are shown in Table III and Table IV, respectively.

TABLE III: IM7/8552: INTRA-LAMINAR FRACTURE PROPERTIES AND PHASE-FIELD PARAMETERS

\begin{tabular}{llllll}
\hline \hline $\mathfrak{G}_{\mathbf{c}, \mathbf{F F}}$ & $\mathfrak{G}_{\mathbf{c}, \mathbf{I F F}}$ & $\oint_{\mathbf{F F}}$ & $\oint_{\mathbf{I F F}}$ & $\boldsymbol{\xi}_{\mathbf{F F}}$ & $\boldsymbol{\xi}_{\mathbf{I F F}}$ \\
$(\mathrm{N} / \mathrm{mm})$ & $(\mathrm{N} / \mathrm{mm})$ & $(\mathrm{mm})$ & $(\mathrm{mm})$ & $(-)$ & $(-)$ \\
\hline 81.5 & 0.2774 & 0.273 & 0.07 & 50 & 50 \\
\hline \hline
\end{tabular}

TABLE IV: E-GLASS/MY750: INTRA-LAMINAR FRACTURE PROPERTIES AND PHASE-FIELD PARAMETERS

\begin{tabular}{llllll}
\hline \hline $\mathfrak{G}_{\mathbf{c}, \mathbf{F F}}$ & $\mathfrak{G}_{\mathbf{c}, \mathbf{I F F}}$ & $\oint_{\mathbf{F F}}$ & $\oint_{\mathbf{I F F}}$ & $\xi_{\mathbf{F F}}$ & $\xi_{\mathbf{I F F}}$ \\
$(\mathrm{N} / \mathrm{mm})$ & $(\mathrm{N} / \mathrm{mm})$ & $(\mathrm{mm})$ & $(\mathrm{mm})$ & $(-)$ & $(-)$ \\
\hline 64 & 1.8 & 0.19 & 1.9 & 50 & 50 \\
\hline \hline
\end{tabular}

In the third application studying the delamination migration in the multi-layered UFRPs, Cohesive Layers (CLs) are added. The properties of the CL in accordance with [19] are listed in Table III.

TABLE V: COHESIVE LAYER PROPERTIES

\begin{tabular}{lllll}
\hline \hline & \multicolumn{5}{c}{ Nomina } \\
$\begin{array}{l}\text { Nominal } \\
\text { strength } \\
(\mathrm{MPa})\end{array}$ & $\begin{array}{l}\text { Nominal } \\
\text { strength in } \\
\text { shear }(\mathrm{MPa})\end{array}$ & $\begin{array}{l}\text { l } \\
\text { fracture } \\
\text { energy } \\
(\mathrm{N} / \mathrm{mm})\end{array}$ & $\begin{array}{l}\text { fracture } \\
\text { energy in } \\
\text { shear }(\mathrm{N} / \mathrm{mm})\end{array}$ & $\begin{array}{l}\text { Power }(\mathrm{BK} \\
\text { law) }\end{array}$ \\
\hline 15 & 15 & 0.5 & 0.65 & 0.43 \\
\hline \hline
\end{tabular}


initiated and propagated along the $90^{\circ} / 0^{\circ}$ interfaces. The occurrence of such matrix cracking events follows the expected sequence inline with [21], see Figs. 2(b)-(f).

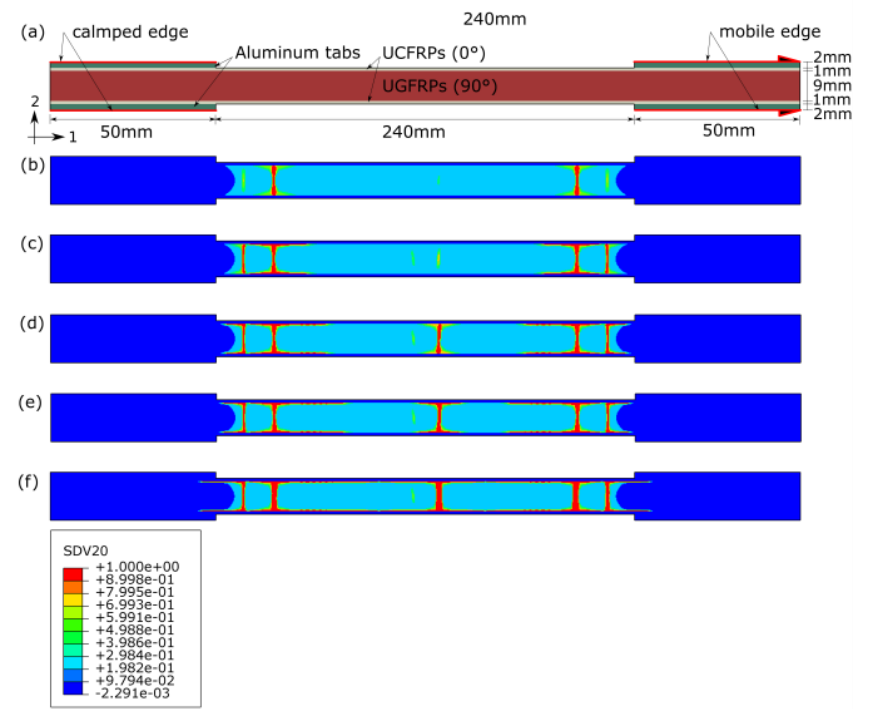

Fig. 2. Matrix Cracking in a Cross-Ply Laminate: (a) Specimen Geometry and Boundary Conditions and (b), (c), (d), (e), and (f) Inter-Fiber Failure Phase-Field Parameter Evolution (SDV20).

\section{Delamination Migration in a Multi-Layered Laminate}

In Fig. 2, the configuration under investigation is depicted. It consists of 44 cross-ply IM7/8552 laminates with the layup sequence $\left[90_{4}^{\circ} / 0_{3}^{\circ} /\left(90^{\circ} / 0^{\circ}\right)_{2 \mathrm{~s}} / 0_{3}^{\circ} /\right.$

$\mathrm{CL} / 90_{4}^{\circ} / \mathrm{CL} / 0_{2}^{\circ} /$ Each ply has a

$\left.\left(90^{\circ} / 0^{\circ}\right)_{2 \mathrm{~s}} / 0_{2}^{\circ} / 90_{3}^{\circ} / 0^{\circ} / 90^{\circ}\right]$

thickness of $0.125 \mathrm{~mm}$. In the numerical model, the PTFE (Polytetrafluoroethylene) layer is replaced by a cohesive layer and in addition, another cohesive layer is added at the interface between the $90_{4}^{\circ}$ and $0^{\circ}$ sequence in order to account for delamination migration. It is obvious from the experimental findings recorded in [20] that migration/kinking occurs as shear stresses in the model shift sign and the crack propagates to the $90_{4}^{\circ}$ layers enabling inter-fiber failure. A 2D analysis is carried with an out-of-plane thickness of $8.37 \mathrm{~mm}$. Hence, the domain is discretized through employing 960000 4-node quadrilateral plane stress elements with an average element size of $0.04 \mathrm{~mm}$, such that each layer of the cross-ply contains at least 4 elements across its thickness. The specimen is loaded under displacement control with constant increments of $\Delta \overline{\mathrm{u}}=2 \times 10^{-3} \mathrm{~mm}$ until the migration is realized.
As was previously discussed, based on postulations made in [20] and the corresponding thorough discussion, delamination migration occurs due to a change of sign in the shear stress components. Negative shearing stresses promote delamination growth at the $0^{\circ} / 90^{\circ}$ interface, and positive shearing stresses promote migration/kinking into $90^{\circ}$ plies. The kinking happens at multiple sites across the specimen. Due to the diffusive nature of the bulk cracks, the shearing stress change can easily be noticed by the initiation of the inter-fiber phase-field as depicted in Fig. 2. Notice that, due to the negative sign at the beginning, delamination propagates until a certain point until shearing stresses are positive. Meanwhile, inter-fiber failure is already initiated, but from the opposite direction, i.e. $90^{\circ} / 0^{\circ}$ interface, but is not nucleated. Whereas, when the shear stresses become positive in the adjacent increments, the migration starts developing, with a crack front now migrating into the $90^{\circ} / 0^{\circ}$ interface.

For a phenomenology of embodiment, in the cohesive layers, once the failure criterion is met, the cohesive layer starts delaminating. Similarly, when the Puck criterion is violated, the inter-fiber failure phase-field is activated due to shearing stresses in the model. As long as the $Q_{\text {IFF }}$ is active, the inter-fiber failure phase-field crack $d_{\text {IFF }}$ grows and migrates into the $90^{\circ}$ plies until the $90^{\circ} / 0^{\circ}$ interface. Simultaneously, the top cohesive layer at the $90^{\circ} / 0^{\circ}$ interface starts to delaminate. When the migration crack front crosses the $90^{\circ} / 0^{\circ}$ interface, the crack front is again propagating due to the negative shear stress leading to the delamination of the top cohesive layer. Here onward, crack propagation is dominated by the residual stresses.

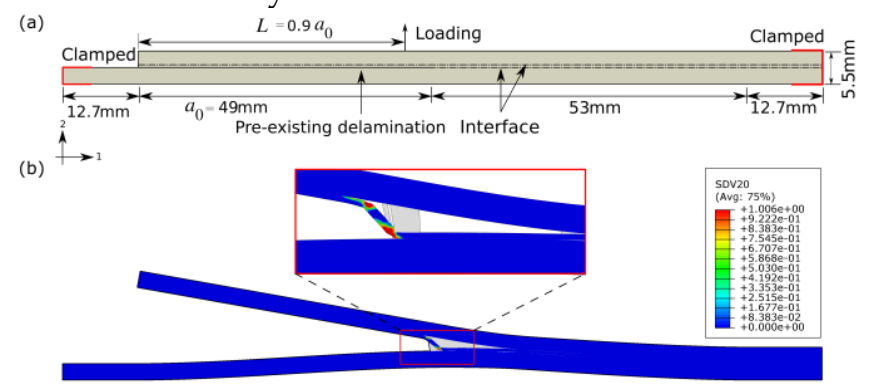

Fig. 3. Delamination Migration: (a) Specimen Geometry and Boundary Conditions and (b) Inter-Fiber Failure Phase-Field Parameter Evolution (SDV20). 


\section{CONCLUSION}

Fracture events in different Unidirectional Fiber Reinforced Polymers (UFRPs) laminates were investigated virtually using the Finite Element (FE) code ABAQUS employing a newly proposed multi Phase-Field (PF) fracture model [1]. Three different fracture problems were herein considered (i) fracture in single-edge notched laminated specimens, (ii) matrix cracking in cross-ply laminates, and (iii) delamination migration in multi-layered UFRPs. In all the presented cases, the predicted fracture events along with their evolution were inline with expected outcomes for such cases, evidencing the reliability and predictive capability of the employed model.

\section{ACKNOWLEDGMENT}

The authors would like to thank Marco Paggi and Christian Gerendt, who contributed to the success of this work through numerous expert discussions.

\section{REFERENCES}

[1] A. Dean, P. Kumar, J. Reinoso, C. Gerendt, M. Paggi, E. Mahdi, et al., "A multi phase-field fracture model for long fiber reinforced composites based on the puck theory of failure," Composite Structures, pp. 112446, 2020.

[2] International Handbook of FRP Composites in Civil Engineering; 1st ed., CRC Press, 2013.

[3] A. Dean, N. Safdar, and R. Rolfes, "A co-rotational based anisotropic elasto-plastic model for geometrically non-linear analysis of fibre reinforced polymer composites: Formulation and finite element implementation," Materials, vol. 12, pp. 1816, 2019.

[4] T. Belytschko and T. Black, "Elastic crack growth in finite elements with minimal remeshing," International Journal for Numerical Methods in Engineering, vol. 45, pp. 601-620, 1999.

[5] J.C. Simo, J. Oliver, and F. Armero, "An analysis of strong discontinuities induced by strain-softening in rate-independent inelastic solids," Computational Mechanics, vol. 12, pp. 277-296, 1993.

[6] J. Mosler and G. Meschke, "3d modelling of strong discontinuities in elastoplastic solids: fixed and rotating localization formulations," International Journal for Numerical Methods in Engineering, vol. 57, pp. 1553- 1576, 2003.

[7] J. Y. Wu, V. P. Nguyen, C. T. Nguyen, D. Sutula, S. Sinaie, and S. Bordas, "Phase field modelling of fracture," Advances in Applied Mechanics, 2019.

[8] L. Ambrosio and V. M. Tortorelli, "Approximation of functionals depending on jumps by elliptic functionals via g-convergence," Communications on Pure and Applied Mathematics, vol. 43, pp. 999- 1036, 1990.

[9] G. A. Francfort and J. J. Marigo, "Revisiting brittle fracture as an energy minimization problem," Journal of the Mechanics and Physics of Solids, vol. 46, pp. 1319 - 1342, 1998.

[10] B. Bourdin, G. A. Francfort, and J. J. Marigo, "Numerical experiments in revisited brittle fracture," Journal of the Mechanics and Physics of Solids, vol. 48, pp. 797- 826, 2000.
[11] B. Bourdin, G. A. Francfort, and J. J. Marigo, "The variational approach to fracture," Journal of Elasticity, vol. 91, pp. 5- 148, 2008

[12] A. Dean, N. Grbic, R. Rolfes, and A. Behrens, "Macro-mechanical modeling and experimental validation of anisotropic, pressure- and temperature-dependent behavior of short fiber composites," Composite Structures, vol. 211, pp. 630- 643, 2019.

[13] A. Dean, S. Sahraee, K. Ozenc, J. Reinoso, R. Rolfes, and M. Kaliske, "A thermodynamically consistent framework to couple damage and plasticity microplane-based formulations for fracture modeling: development and algorithmic treatment," International Journal of Fracture, vol. 203, pp. 115-134, 2016.

[14] M. Deuschle and B. H. Kröplin, "Finite element implementation of puck's failure theory for fibre-reinforced composites under three-dimensional stress," Journal of Composite Materials, vol. 46, pp. 2485- 2513, 2012.

[15] M. J. Borden, T. J. R. Hughes, C. M. Landis, and C. V. Verhoosel, “A higher-order phase-field model for brittle fracture: Formulation and analysis within the isogeometric analysis framework," Computer Methods in Applied Mechanics and Engineering, vol. 273, pp. 100118,2014

[16] A. Dean, J. Reinoso, N.K. Jha, E. Mahdi, and R. Rolfes, "A phase field approach for ductile fracture of short fibre reinforced composites," Theoretical and Applied Fracture Mechanics, vol. 106, pp. 102495, 2014.

[17] A. S. Kaddour and M. Hinton, "Input data for test cases used in benchmarking triaxial failure theories of composites," Journal of Composite Materials, vol. 46, pp. 2295- 2312, 2012.

[18] A. Quintanas-Corominas, J. Reinoso, E. Casoni, A. Turon, and J. A. Mayugo, "A phase field approach to simulate intralaminar and translaminar fracture in long fiber composite materials," Computer Methods in Applied Mechanics and Engineering, vol. 358, pp. 112618, 2020.

[19] X. Li and J. Chen. Mayugo, "A highly efficient prediction of delamination migration in laminated composites using the extended cohesive damage model," Composite Structures, vol. 160, pp. 712 721, 2017.

[20] M. F. Pernice, N. V. De Carvalho, J. G. Ratcliffe, and S. R. Hallett, "Experimental study on delamination migration in composite laminates," Composites Part A: Applied Science and Manufacturing, vol. 73, pp. 20- 43, 2015.

[21] P. Maimí, P. P. Camanho, J. A. Mayugo, and A. Turon, "Matrix cracking and delamination in laminated composites. Part I: Ply constitutive law, first ply failure and onset of delamination," Mechanics of Materials, vol. 43, pp. 169-185, 2011.

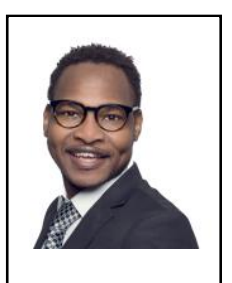

Aamir Dean is a visiting professor at the Elasticity and Strength of Materials Group, University of Seville, Spain. Before that, he was leading the research group 'Fatigue' at the Institute of Structural Analysis, Leibniz University Hannover, Germany. He received two doctorate degrees, in Mechanical Engineering and Industrial Management in 2020 from the University of Seville, Spain, and also in Civil Engineering from the Leibniz University Hannover, Germany in 2017. He is active in the field of computational-, multi-physics-, inelastic-modeling of heterogeneous and anisotropic materials, with a particular interest in polymer composites. In recognition of his research achievements, he has been awarded the Hannover Award of Science, Germany, and the Chamber of Engineers Prize, Germany.

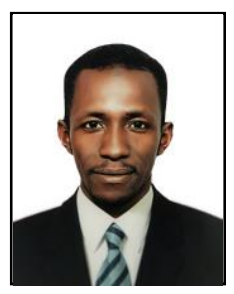

Ammar Babiker received a B.Sc. in civil engineering from Sudan University of Science and Technology, Sudan in 2010, and an M.Sc. in Advanced Computational and Civil Engineering Structural Studies from TU Dresden, Germany in 2017. Currently, he is a Ph.D. and candidate at the Institute of Concrete Structures of the TU Dresden. Since 2017, he has been appointed as a lecturer at Sudan University of Science and Technology. Ammar is now associated as a researcher at the Institute of Concrete Structure of the TU Dresden. His main research interests are fiber-reinforced cement-based composites, material modeling, impact analysis, and strain-rate effect in concrete and cement-based composites. 